\title{
Primary Intraocular Malignant Rhabdoid Tumor Mimicking Retinoblastoma in a Child
}

\author{
Nirupama Kasturia Pratik Gera ${ }^{a}$ Gayatri Panicker ${ }^{a}$ Ajax Jossy ${ }^{a}$

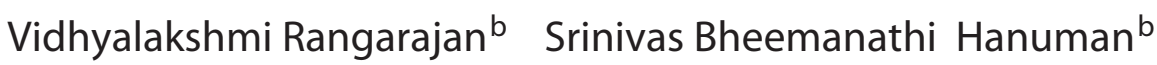 \\ ${ }^{a}$ Department of Ophthalmology, Jawaharlal Postgraduate Medical Education and Research, Puducherry, India; \\ b Department of Pathology, Jawaharlal Postgraduate Medical Education and Research, Puducherry, India
}

\section{Established Facts}

- Malignant rhabdoid tumors occur in the renal, extrarenal soft tissue and central nervous system.

- Most of these tumors share inactivation of the SMARCB1/INI-1 gene on chromosome 22q.

\section{Novel Insights}

- Malignant rhabdoid tumors can rarely occur intraocularly as a primary tumor or as a metastasis from the kidney.

- Overall, 2-3\% of tumors with rhabdoid histology retain expression of the SMARCB1 protein on immunohistochemistry and do not display inactivating mutations in the gene.

\section{Keywords}

Rhabdoid tumor · Leukocoria · Retinoblastoma

\section{Abstract}

Primary rhabdoid tumors are highly malignant, rare tumors occurring in the renal, extrarenal soft tissue or central nervous system. They have non-specific radiological features and present with several histological components that create a problem in differential diagnosis with other embryonal tumors. We report a rare case of malignant rhabdoid tumor of the retina that presented with clinical features like those of retinoblastoma.

(c) 2020 S. Karger AG, Basel

\section{Case}

A 4-year-old male child, born of non-consanguinity, was brought to the ophthalmology clinic by his parents with a history of noticing a white reflex in his left eye for 1 week. It was not associated with any redness, pain, squinting, or photophobia. There was no history of ocular trauma. He was born at term and was developmentally normal. The child was stable, and a systemic examination was unremarkable. Ocular examination showed orthotropia with a normal range of extraocular movements. A white reflex was noted in the left eye. His visual acuity was 6/9 in the right eye and perception of light with an inaccurate projection of rays in the left eye. The anterior segment examination was normal in the right eye. The left eye showed a normal anterior chamber with a briskly reacting pupil and leukocoria (Fig. 1a, b). The fundus examination was normal in the right eye and showed a yellowish karger@karger.com

(c) 2020 S. Karger AG, Basel

www.karger.com/oop

Karger
Nirupama Kasturi

Department of Ophthalmology JIPMER

Puducherry 605006 (India)

kasturiniru@gmail.com 
Fig. 1. a Clinical photograph of the child showing leukocoria in the left eye. b Slitlamp image showing a retrolental yellowish mass with intrinsic vascularity. c Ultrasound B scan showing a hyperechoic mass in the vitreous cavity with calcification. d Coronal and axial MRI scan showing a large intraocular tumor in the vitreous cavity.

Fig. 2. a Gross examination of the left eye enucleation specimen showed an exophytic, gray-white, solid tumor located in the vitreous cavity. b Scanner view photomicrograph revealed a tumor arising from the retinal layer of the eyeball. HE, $\times 40$. c Tumor cells arranged in nests and sheets separated by delicate fibrovascular septae. HE, $\times 100$. d Tumor cells exhibiting a rhabdoid morphology with eccentrically placed round nuclei, vesicular chromatin with intranuclear inclusions in some cells, and an abundant amount of bright eosinophilic cytoplasm. HE, $\times 400$.
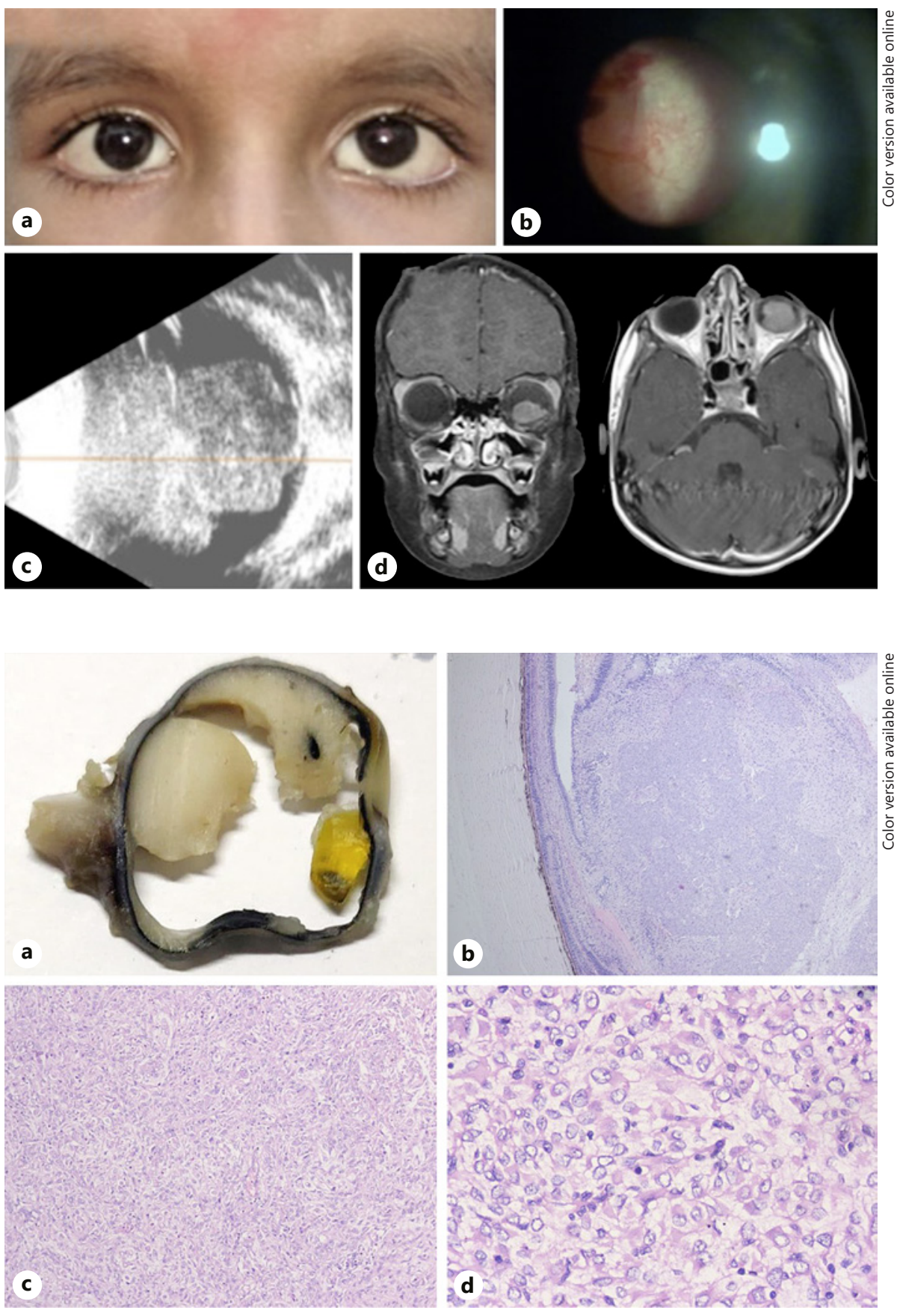

mass with intrinsic vascularity, occupying the vitreous cavity and extending up to the posterior surface of the lens. The optic nerve and macula were not demarcated. An ultrasound B-scan showed a hyperechoic mass lesion occupying almost two thirds of the vitreous cavity extending from the retina to the anterior vitreous (almost touching the lens). Moderate- to high-intensity A-scan spikes were noted with hyperechoic foci inside the mass suggestive of calcification (Fig. 1c). The complete hemogram, chest X-ray, ultrasound abdomen, and whole-body positron emission tomography scan were within normal limits. A brain MRI showed a heteroge- neously enhancing lesion involving the inferior quadrants of the left eye globe with no obvious extrascleral or optic nerve extension (Fig. 1d).

The patient was provisionally diagnosed with group E retinoblastoma and underwent enucleation with orbital implantation under general anesthesia. Histopathology revealed nests and sheets of tumor cells with round to oval vesicular nuclei with fine chromatin, a few of them showing prominent nucleoli, and moderate to abundant pale eosinophilic cytoplasm. Mitotic figures were frequent, around $4-5$ per 10 high-power field (Fig. 2a-d), and calci- 
Fig. 3. On IHC, tumor cells were diffusely positive with vimentin $(\mathrm{DAB}, \times 100$; a) as well as CD99 $(\mathrm{DAB}, \times 200$; b). They were focally positive for IHC with synaptophy$\sin (\mathrm{DAB}, \times 200$; c) and GFAP $(\mathrm{DAB}, \times 400$; d). The cells showed retained expression of INI-1 (DAB, $\times 200$; e) and were negative for desmin, EMA, and S100 (DAB, $\times 100 ; \mathbf{f})$.
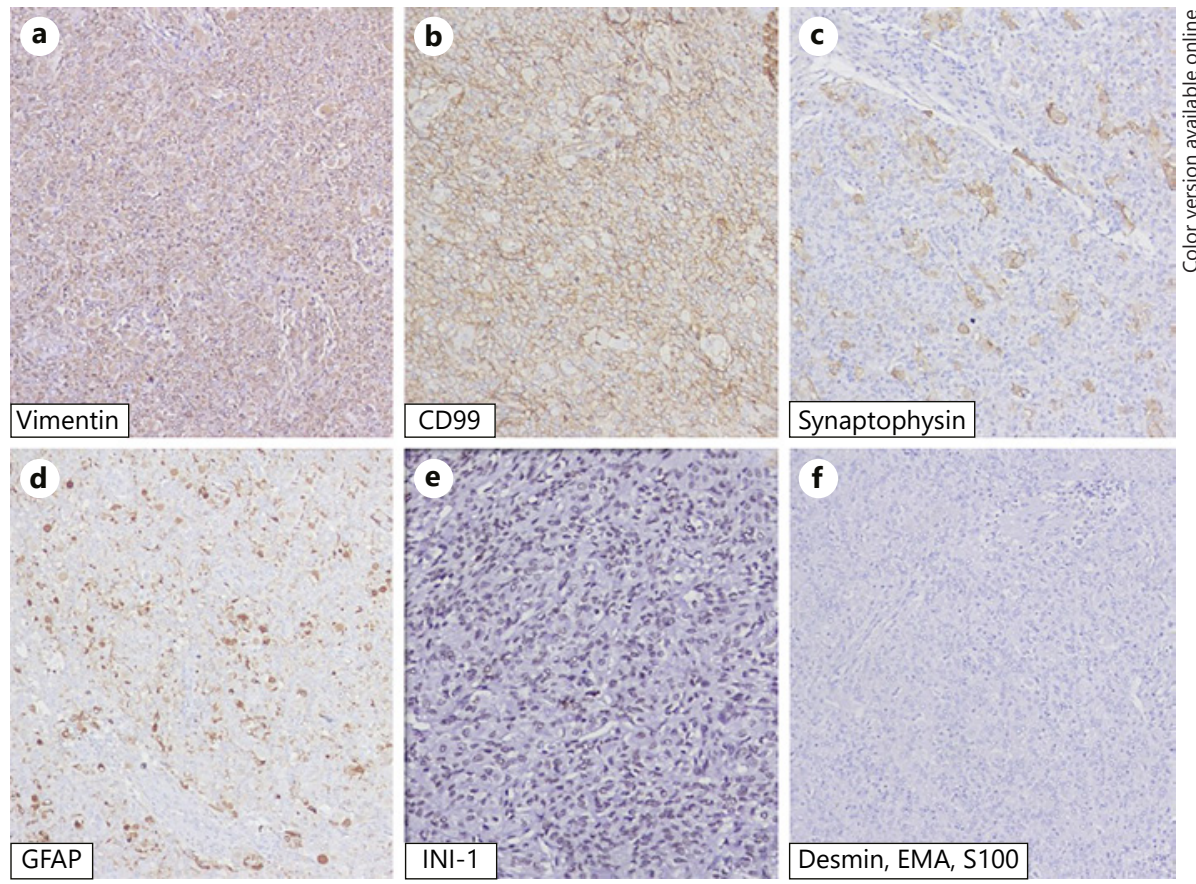

fication was seen focally. On immunohistochemistry (IHC), the tumor cells were diffusely positive for vimentin, pan-cytokeratin, neuron-specific enolase, and CD99, focally positive for glial fibrillary acidic protein (GFAP), synaptophysin, chromogranin, and FLI-1, and negative for EMA, PR, and S100 (ruling out meningioma). The tumor cells showed retained expression of inactivation of the integrase interactor (INI-1) and the Ki-67 index was $12 \%$. The overall histomorphological and immunohistochemical profile favored the possibility of an embryonal tumor with rhabdoid features (Fig. 3a-f). Genetic testing for SMARB1 and RB1 mutation were negative. A heterozygous missense variation in exon 9 of the GUCY2D (chromosome 17) was detected which did not correlate with the clinical findings. The child received six cycles of chemotherapy and is on regular follow-up with no evidence of residual tumor.

\section{Discussion}

Malignant rhabdoid tumors are extremely aggressive childhood neoplasms that have a poor clinical prognosis and occur in the renal, extrarenal soft tissue location, and the central nervous system (atypical teratoid/rhabdoid tumor) [1]. The "rhabdoid" cells are large epithelioid cells with an eosinophilic cytoplasm, eccentric vesicular nuclei, prominent nucleoli, and globoid or fibrillar paranuclear inclusions corresponding ultra-structurally to whorled bundles of intermediate filaments [2]. These tumors usually develop in infants and young children, occur sporadically or as part of rhabdoid tumor predisposition syn- drome, and share inactivation of the SMARCB1/INI-1 gene on chromosome 22q (a classic tumor suppressor gene) $[3,4]$. However, germline perturbations in SMARCB1 is estimated to occur only in approximately $25-30 \%$ of patients with malignant rhabdoid tumors and in our case there were no variations in the gene. The INI-1 expression was retained by the tumor nuclei, which is extremely unusual for this tumor type. In a study by Perry et al. [2], composite rhabdoid tumors derived secondarily from other tumor types like carcinoma, melanoma, sarcoma, or meningioma showed retained expression of INI-1. These are more common in adults, less aggressive, and the rhabdoid cytology represents a common end-stage pattern in these histogenetically diverse groups of tumors. Malignant rhabdoid tumor has been reported in the ocular region secondarily as an ocular metastasis from the renal tumor or primarily in the orbit, retina, and optic nerve [5-8].

MRI often shows an isointense or hypointense signal on T1- and T2-weighted images with areas of hemorrhage, calcification, necrosis, or edema [9]. The differential diagnosis includes medulloepitheliomas, primitive neuroectodermal, and germ cell tumors. Medulloepitheliomas arise in the ciliary body region, contain intratumoral cysts, and a histopathological appearance comprising of primitive neuroepithelial cells surrounded by a mucopolysaccharide-rich matrix. The presence of perinuclear cytoplasmic inclusions is usually more characteristic of rhabdoid tumors and eccentrically placed nuclei 
with prominent central nucleoli resembling rhabdomyoblasts, while the neoplastic cells show skeletal muscle differentiation in the form of cross-striations and strap cells in rhabdomyosarcoma. Malignant rhabdoid tumor shares some morphological features with those entities, but the presence of rhabdoid cells and IHC helps to establish the diagnosis. For treatment, there are no definitive guidelines for malignant rhabdoid tumors because of their rarity. However, it is based on surgical resection, chemotherapy, and radiotherapy [10].

\section{Conclusion}

Primary intraocular malignant rhabdoid tumors are overly aggressive embryonal tumors that can rarely mimic retinoblastoma. An accurate diagnosis is possible with their histomorphologic features and IHC. Neuroimaging to look for a synchronous or metachronous central nervous system tumor is essential.

\section{Acknowledgements}

The authors acknowledge the contribution of Dr. Amit Kumar Deb, Dr. Mary Stephen, Dr. Pragathi Sparsh, and Dr. Vishnu Nair in the clinical management of this patient.

\section{Statement of Ethics}

Written informed consent for publication (including the images) has been obtained from the parent. All procedures carried out were in accordance with the tenets of the Declaration of Helsinki. Institutional ethics committee approval is not required for a case report according to the Indian council of medical research guidelines.

\section{Conflict of Interest Statement}

The authors declare no conflicts of interest for this case study.

\section{Funding Sources}

Funding was received from CanKids fundraising organisation for the histopathological and molecular testing performed in this study.

\section{Author Contributions}

N.K., P.G., G.P., and A.J. contributed to the patient evaluation, management, and follow-up. S.B.H. and V.R. provided the histopathological details. All authors contributed to the manuscript preparation and review.

\section{References}

1 Biggs PJ, Garen PD, Powers JM, Garvin AJ. Malignant rhabdoid tumor of the central nervous system. Hum Pathol. 1987 Apr;18(4):332-7.

2 Perry A, Fuller CE, Judkins AR, Dehner LP, Biegel JA. INI1 expression is retained in composite rhabdoid tumors, including rhabdoid meningiomas. Mod Pathol. 2005 Jul;18(7): 951-8.

3 Judkins AR, Eberhart CG, Wesseling P. Atypical teratoid/rhabdoid tumour. World Health Organization Classification of tumours of the central nervous system. Lyon: IARC Press; 2007. pp. 147-9.

4 Sigauke E, Rakheja D, Maddox DL, Hladik CL, White CL 3rd, Timmons CF, et al. Absence of expression of SMARCB1/INI1 in ma- lignant rhabdoid tumors of the central nervous system, kidneys and soft tissue: an immunohistochemical study with implications for diagnosis. Mod Pathol. 2006 May;19(5): $717-25$.

5 Verma A, Morriss C. Atypical teratoid/rhabdoid tumor of the optic nerve. Pediatr Radiol. 2008 Oct;38(10):1117-21.

6 Shah SJ, Ali MJ, Mulay K, Honavar SG, Reddy VA. Primary intraocular malignant extrarenal rhabdoid tumour: a clinicopathological correlation. J Pediatr Ophthalmol Strabismus. 2013 Apr;50:e18-20.

7 Ayala Barroso E, Tapia Bahamondes A, Sánchez España JC, Alós L, Medel Jiménez R. Primary intraocular malignant rhabdoid tumor without extrascleral compromise. J Pediatr Ophthalmol Strabismus. 2018 Apr;55:e7-9.

8 Mahdi Y, Kharmoum J, Alouan A, Elouarradi H, Elkhiyat I, Maher M, et al. Primary atypical teratoid/rhabdoid tumor of the optic nerve: a rare entity in an exceptional location. Diagn Pathol. 2015 May;10(1):47.

9 Parmar H, Hawkins C, Bouffet E, Rutka J, Shroff M. Imaging findings in primary intracranial atypical teratoid/rhabdoid tumors. Pediatr Radiol. 2006 Feb;36(2):126-32.

10 Hada M, Chawla B, Seth R, Khurana S, Kashyap S. Primary intraocular malignant rhabdoid tumor presenting as orbital mass with intracranial extension in an adolescent. Can J Ophthalmol. 2017 Feb;52(1):e3-5. 